\title{
An Empirical Study of Slice-Based Cohesion and Coupling Metrics
}

\author{
Timothy M. Meyers and David Binkley \\ Loyola College in Maryland \\ Baltimore, Maryland \\ 21210-2699, USA \\ \{tmeyers,binkley\}@cs.loyola.edu
}

\begin{abstract}
Software reengineering is a costly endeavor, due in part to the ambiguity of where to focus reengineering effort. Coupling and Cohesion metrics, particularly quantitative cohesion metrics, have the potential to aid in this identification and to measure progress. The most extensive work on such metrics is with slice-based cohesion metrics. While their use of semantic dependence information should make them an excellent choice for cohesion measurement, their wide spread use has been impeded in part by a lack of empirical study.

Recent advances in software tools make, for the first time, a large-scale empirical study of slicebased cohesion and coupling metrics possible. Four results from such a study are presented. First, "head-to-head" qualitative and quantitative comparisons of the metrics identify which metrics provide similar views of a program and which provide unique views of a program. This study includes statistical analysis showing that slice-based metrics are not proxies for simple size-based metrics such as lines of code. Second, two longitudinal studies show that slice-based metrics quantify the deterioration of a program as it ages. This serves to validate the metrics: the metrics quantify the degradation that exists during development; turning this around, the metrics can be used to measure the progress of a reengineering effort. Third, base-line values for slicebased metrics are provided. These values act as targets for reengineering efforts with modules having values outside the expected range being the most in need of attention. Finally, slice-based coupling is correlated and compared with slice based cohesion.
\end{abstract}

Categories and Subject Descriptors: D.2.8 [Software Engineering]: Metrics; D.2.2 [Software Engineering]: Design Tools and Techniques; D.2.3 [Software Engineering]: Coding Tools and Techniques

General Terms: Reengineering, Software Intervention

Additional Key Words and Phrases: Slicing, Cohesion, Coupling

\section{INTRODUCTION}

The goal of a cohesion metric is to give a quantitative estimate of the level of cohesion in a module. In principal, good cohesion metrics help guide software intervention by revealing modules within a program that are in need of reconstruction. These poor quality modules have higher construction, testing, maintenance, and comprehension costs. Two important prerequisites to this identification are (1) knowing that the metrics provide a sufficient quantitative measure of cohesion (and ideally module quality), and (2) knowing which metric values indicate normality and which indicate a problem.

Lakhotia differentiates between definitions of cohesion that are subjective and thus not automateable and those that are functional and thus can be computed by a rule based approach [Lakhotia and Sterling 1988]. Most prior work on cohesion metrics has studied qualitative cohesion metrics [Constantine and Yourdon 1979]. Limited work on quantitative cohesion metrics has been undertaken. For example, Bieman et al. developed and formalized several quantitative cohesion metrics [Bieman and Ott 1994; Ott and Thuss 
1993]. To date, however, little empirical investigation of these metrics has been performedprimarily due to insufficient tool support. Empirical data can potentially provide valuable evidence (hopefully) tying the metrics to code cohesion and quality.

Assuming the existence of such a relationship, one could sort the modules of a program based on their metric values and consider restructuring those modules below some arbitrary cutoff (e.g., those in the bottom $10 \%$ of the sorted list). However, this inevitably leads to spending too much effort on "good" programs and too little effort on "bad" programs. A better approach would be to know which metric values are "normal" and which indicate the need for intervention. Then only those modules whose metric values were outside the normal range would be considered for restructuring.

The most extensive research on quantitative cohesion measurement is the work of Bieman and Ott [Bieman and Ott 1994; Ott and Thuss 1993]. Their cohesion metrics are based on program slicing: a program simplification technology that removes from a program components (e.g., statements) that do not affect a computation of interest [Weiser 1981; Binkley and Gallagher 1996]. The resulting (smaller) program, called a slice, captures a projection of the semantics of the original program [Reps and Yang 1988; Binkley et al. 1995].

Slice-based metrics, introduced more than two decades ago, exploit the semantic relationships that underlie slicing. To date, however, they have lacked intensive empirical investigation due to a lack of mature tools. For example, while many previous studies make use of slice-based metrics [Weiser 1981; Ott and Thuss 1993; 1989; Bieman and Ott 1994; Harman et al. 1995a; Karstu 1994; Bieman and Kang 1998], they all have significant limitations. The most serious of these are the small size and limited number of the programs studied. Other limitations include slicers that do not correctly handle complex language features such as pointer variables, and slicers that ignore certain statement types, for example, declarations, gotos, breaks, etc.

Slicing technology is now mature enough to allow an extensive empirical investigation of slice-based metrics. For example, the deep structure analysis tool, Codesurfer [Grammatech Inc. 2002], used in this study of slice-based cohesion and coupling metrics, handles the complete $\mathrm{C}$ (as well as $\mathrm{C}++$ ) language. Thus, the resulting study overcomes the limitations found in previous studies. It reports metric values for the metrics introduced by Weiser, Bieman and Ott, and Harman [Weiser 1981; Bieman and Ott 1994; Harman et al. 1995b] computed from a code base of 22,651 procedures from 63 programs that range in size from about 500 to over 150,000 lines of code. In all, over 1.7 million lines of code were analyzed with a total of 3,194,479 slices being taken. The results from this study form the paper's three main contributions:

-First, the study compares the slice-based metrics: Tightness, MinCoverage, Coverage, MaxCoverage, and Overlap [Weiser 1981; Ott and Thuss 1993] and slice-based coupling "head-to-head." Both qualitative and quantitative comparisons show which of the metrics are strongly correlated and which provide distinct views of a program. This study also compares slice-based cohesion and coupling metrics with lines of code as a representative for various size-based metrics (e.g., number of functions). The results show that slice-based metrics provide a unique view of a program and are not proxy for size-based metrics.

- Second, perhaps the most important result of this study, is an indication that slice-based metrics quantify overall code quality. Following a suggestion by Ott and Thuss [Ott 
and Thuss 1989], the effect of software evolution on slice-based metrics is measured. Two such longitudinal studies are presented. They seek to quantify the degradation of software quality caused by maintenance and evolution. The results provide evidence that the metrics successfully quantify code quality; thus, they can be used to guide and measure software intervention efforts.

- Third, the study provides base-line values for the slice-based cohesion metrics and slicebased coupling metrics, which has not been well studied [Harman et al. 1997]. Base-line values are useful in the identification of degraded modules. They also aid in the transfer of technology from academia to industry [Zelkowitz et al. 1998; Redwine and Riddle 1985].

The remainder of this paper is organized as follows: Section 2 provides background information. The main results are considered in Section 3. These results are related to prior work in Section 4. Possible threats to the study are presented in Section 5, and finally a summary is provided in Section 6 .

\section{BACKGROUND}

This section provides background material on program slicing, the metrics considered, and finally the statistical tests employed.

\subsection{System Dependence Graph}

A slice can be computed as the solution to a graph reachability problem over a program's System Dependence Graph (SDG) [Horwitz et al. 1990]. An SDG is a collection of Procedure Dependence Graphs (PDGs) connected by interprocedural control- and flowdependence edges. Each PDG contains vertices that represent the components (statements and predicates) of the procedure and edges that represent control dependences, flow dependences, and transitive summary dependences between the vertices. The vertices of a PDG are essentially those of the procedure's control-flow graph. A control dependence edge from vertex $u$ to vertex $v$ means that, during execution, the predicate represented by $u$ controls the execution of $v$. For structured languages, control dependences reflect the program's nesting structure. A PDG contains a flow dependence edge from vertex $u$ defining variable $x$ to vertex $v$ that uses $x$ if control can reach $v$ after $u$ via an execution path along which there is no intervening definition of $x$. Finally, at call sites there are transitive dependence edges that summarize transitive dependences induced by called procedures. These dependences capture the effects of the initial values of parameters and global variable on their values after the call. The dependences in a PDG are safe approximations to the semantic dependences found in the program [Podgurski and Clarke 1990], which are in general not computable and therefore must be approximated.

An SDG is a collection of procedure dependence graphs connected at call-sites by interprocedural control- and flow-dependence edges. In an SDG, a call statement is represented using a call vertex; parameter passing is represented using four kinds of parameter vertices: on the calling side, parameter passing is represented by actual-in and actual-out vertices, which are control dependent on the call vertex; in the called procedure, parameter passing is represented by formal-in and formal-out vertices, which are control dependent on the called procedure's entry vertex.

A slice of a PDG $P$, taken with respect to a set of vertices $S$, contains those vertices of $P$ whose components potentially affect the components represented by vertices in $S$ [Horwitz 


\begin{tabular}{|c|c|c|}
\hline Metric & Definition & Description \\
\hline Tightness $(M)=$ & $\frac{\left|S L_{\text {int }}\right|}{\text { length }(M)}$ & $\begin{array}{l}\text { Tightness measures the number of } \\
\text { statements in every slice. }\end{array}$ \\
\hline $\operatorname{MinCoverage}(M)=$ & $=\frac{1}{\text { length }(M)} \min _{i}\left|S L_{i}\right|$ & $\begin{array}{l}\text { MinCoverage is the ratio of the smallest } \\
\text { slice in a module to the module's length. }\end{array}$ \\
\hline Coverage $(M)=$ & $\frac{1}{\left|V_{O}\right|} \sum_{i=1}^{\left|V_{O}\right|} \frac{\left|S L_{i}\right|}{\operatorname{length}(M)}$ & $\begin{array}{l}\text { Coverage compares the length of slices } \\
\text { to the length of the entire module. }\end{array}$ \\
\hline MaxCoverage $(M)$ & $=\frac{1}{\text { length }(M)} \max _{i}\left|S L_{i}\right|$ & $\begin{array}{l}\text { MaxCoverage is the ratio of the largest } \\
\text { slice in a module to the module's length. }\end{array}$ \\
\hline Overlap $(M)=$ & $\frac{1}{\left|V_{O}\right|} \sum_{i=1}^{\left|V_{O}\right|} \frac{\left|S L_{i n t}\right|}{\left|S L_{i}\right|}$ & $\begin{array}{l}\text { Overlap is a measure of how many } \\
\text { statements are common to all slices. }\end{array}$ \\
\hline Parallelism $(M, \tau)$ & $\begin{aligned}= & |A|, \text { where } A= \\
& \left\{S L_{i} \ni\left|S L_{i} \cap S L_{j}\right| \leq\right. \\
& j \neq i\}\end{aligned}$ & $\begin{array}{l}\text { Parallelism is the number of slices with } \\
\text { no more than } \tau \text { statements in common. }\end{array}$ \\
\hline
\end{tabular}

Fig. 1. The Cohesion Metrics

et al. 1990]. To compute cohesion metrics for each procedure, a slice which includes only vertices from a single procedure is used. This slice can be computed as a simple transitive closure over the dependence edges in a PDG. However, as the slice makes use of the SDG's summary edges, it correctly accounts for the dependence effects of transitively called procedures.

A slice of SDG $G$, taken with respect to a set of vertices $S$, contains those vertices of $G$ whose components potentially affect the components represented in $S$ [Horwitz et al. 1990]. While simple transitive closure is sufficient, the resulting set of components whose vertices have a path to a vertex in $S$ is needlessly large. More precise interprocedural slices can be computed using a two pass algorithm [Horwitz et al. 1990]. Pass 1 starts from all vertices in $S$ and goes backwards (from target to source) along the edges of the SDG without descending into called procedures. Pass 2 starts from all vertices reached in Pass 1 and goes backwards along the edges of the SDG but does not ascend to calling procedures. The resulting slice is the set of vertices encountered during Pass 1 and Pass 2 .

\subsection{Slice-Based Cohesion Metrics}

In his original work on program slicing, Mark Weiser proposed using metrics based on program slicing to measure program cohesion [Weiser 1981]. He informally presented five sliced-based metrics: Tightness, Coverage, Overlap, Parallelism, and Clustering [Weiser 1981]. Of these, Ott and Thuss formalized all but Clustering, observing that it was too vague to properly formalize [Ott and Thuss 1993]. Therefore, Clustering is not considered herein. Additionally, Parallelism, has not proven useful in software engineering. One 
reason for this is that it is considerably more complex and therefore less intuitive than the other metrics. While in theory, higher values of Parallelism indicate that a module contains multiple unrelated or only slightly related threads (suggesting it be targeted for reconstruction), other slice-based metrics provide similar information and are significantly easier to comprehend; thus, for completeness, Parallelism is investigated separately.

The formalization of the remaining metrics is done in terms of the variables $V_{M}$, the set of variables used by module $M$ (the term module refers to the unit of code being considered, herein a $\mathrm{C}$ function). $V_{O}$, the subset of $V_{M}$ that are output variables (call principal variables in some metrics work [Bieman and Ott 1994; Harman et al. 1997]). In the study, $V_{O}$ contains a function's return value and those globals modified by the function. Finally, $S L_{i}$ is the slice obtained for $v_{i} \in V_{O}$ and $S L_{i n t}$, the intersection of $S L_{i}$ over all $v_{i} \in V_{O}$.

Figure 1 shows Weiser's metrics (except Clustering), and MinCoverage and MaxCoverage, two additional cohesion metrics proposed by Ott and Thuss [Ott and Thuss 1993]. The figure also provides an informal description of each metric. Note that with the exception of Parallelism, metric values range from 0.0 to 1.0.

EXAMPLE. Figure 2 illustrates the computation of these metrics using the example program shown in Figure 3. For the purpose of this example, $V_{O}$ contains smallest and largest, the two variables output by print $f$ statements. Intuitively, Tightness favors concise single thought modules where most of the statements affect all of the outputs (i.e., are in $S L_{\text {int }}$ ). By construction, the example produces a fairly high value of Tightness with almost two-thirds of the statements being in both slices. Weiser notes that a low value of Coverage may be indicative of a module with several distinct conceptual purposes [Weiser 1981]. In comparison with Tightness, Coverage also favors large slices, but does not require them to overlap and thus does not require as high a degree of "single thoughtness". At one extreme, a module that divides into two independent slices has a Coverage of 0.5, but a Tightness of 0.0. At the other extreme, higher values of Coverage force increases in the size of $S L_{\text {int }}$; thus, a Coverage of 1.0 implies a Tightness of 1.0 and vise versa. In the example, the inclusion of statements not in $S L_{\text {int }}$ such as the printfs accounts for Coverage's higher value. Informally, MaxCoverage considers if a module includes at least one particularly long slice and thus is dominated by a least one computation. Symmetrically, MinCoverage considers if a module includes at least one particularly short slice and thus includes some (perhaps unwanted) side computation. Finally, for Overlap consider a module that includes a main computation and then derives $k$ results from this computation each in a single statement. As $k$ increases both Tightness and Coverage decrease (because the length of the module is increasing). Overlap, however, remains constant reflecting that all the $k$ results share the same main computation.

\subsection{Slice-Based Coupling}

In general, coupling between modules or components is their degree of mutual interdependence. Traditionally, when measured, coupling is calculated in terms of Henry and Kafura's information flow metrics [Henry and Kafura 1981], which are defined in terms of the total information flowing in to a module (inflow) and the total information flowing out of a module (outflow). Essentially, inflow captures definitions of variables from outside a module whose values are used by the module, and outflow captures definitions from within the module whose values are used in other modules.

In general, lower coupling is better. Page-Jones provide three motivations for this [PageJones 1980]: (1) fewer interconnections between modules reduce the chance that a fault 


\begin{tabular}{|llr|}
\hline \multicolumn{1}{|c}{ Metric } & \multicolumn{1}{c|}{ Computation } & Value \\
\hline Tightness & $=11 / 19$ & $=0.58$ \\
MinCoverage & $=14 / 19$ & $=0.74$ \\
Coverage & $=1 / 2(14 / 19+16 / 19)=15 / 19$ & $=0.79$ \\
MaxCoverage & $=16 / 19$ & $=0.84$ \\
Overlap & $=1 / 2(11 / 14+11 / 16)=$ & $=0.74$ \\
Parallelism & $=0$ for $\tau \leq 11$ and 1 otherwise & \\
\hline
\end{tabular}

Fig. 2. Example Metrics Computations

\begin{tabular}{|c|c|c|c|}
\hline Program & $S L_{\text {smallest }}$ & $S L_{\text {largest }}$ & $S L_{\text {int }}$ \\
\hline \multicolumn{4}{|l|}{$\operatorname{main}()$} \\
\hline \multicolumn{4}{|l|}{ int i; } \\
\hline \multirow{2}{*}{\multicolumn{4}{|c|}{$\begin{array}{l}\text { int smallest; } \\
\text { int largest; }\end{array}$}} \\
\hline & & & \\
\hline \multicolumn{4}{|l|}{ int $\mathrm{A}[10]$} \\
\hline \multicolumn{4}{|l|}{ for $(\mathrm{i}=0 ; \mathrm{i} ; 10 ; \mathrm{i}++)$} \\
\hline \multirow{2}{*}{\multicolumn{4}{|c|}{$\begin{array}{l}\text { int num; } \\
\text { scanf("\%d", \&num); }\end{array}$}} \\
\hline & & & \\
\hline \multicolumn{4}{|l|}{$\mathrm{A}[\mathrm{i}]=$ num; } \\
\hline \multicolumn{4}{|l|}{\}} \\
\hline \multirow{2}{*}{\multicolumn{4}{|c|}{$\begin{array}{l}\text { smallest = A[0]; } \\
\text { largest = smallest; }\end{array}$}} \\
\hline & & & \\
\hline \multirow{2}{*}{\multicolumn{4}{|c|}{$\begin{array}{l}\mathrm{i}=1 \\
\text { while }(\mathrm{i}<10)\end{array}$}} \\
\hline & i & | & | \\
\hline \multirow{3}{*}{\multicolumn{4}{|c|}{$\begin{array}{l}\text { if }(\text { smallest }>\mathrm{A}[\mathrm{i}]) \\
\text { smallest }=\mathrm{A}[\mathrm{i}]\end{array}$}} \\
\hline & & & \\
\hline & & & \\
\hline \multirow{2}{*}{\multicolumn{4}{|c|}{$\begin{array}{l}\text { if (largest }<A[\mathrm{i}] \text { ) } \\
\text { largest }=\mathrm{A}[\mathrm{i}]\end{array}$}} \\
\hline & & & \\
\hline $\mathrm{i}=\mathrm{i}+1$ & | & | & | \\
\hline \multicolumn{4}{|l|}{\}} \\
\hline \multirow{2}{*}{\multicolumn{4}{|c|}{$\begin{array}{l}\operatorname{printf("\circ d~} \backslash n ", \text { smallest); } \\
\text { printf("\%d } \backslash n ", \text { largest); }\end{array}$}} \\
\hline & & & \\
\hline & & & \\
\hline length: 19 & 14 & 16 & 11 \\
\hline
\end{tabular}

Fig. 3. An example program. Vertical bars in the last three columns indicate membership in the slice taken with respect to the output of smallest, the output largest, and their intersection, respectively.

in one module will cause a failure in other modules, (2) fewer interconnections between modules reduce the chance that changes in one module cause problems in other modules, which enhances reusability, and (3) fewer interconnections between modules make it easier for developers to understand details of other modules. Offutt et al. cite several research projects that have studied coupling [Li and Offutt 1994]. For example, they observe that changing tightly coupled modules requires more effort than changing modules that are loosely coupled to each other.

ACM Transactions on Software Maintenance, Vol. V, No. N, November 2007. 
Harman et al. in a short position paper [Harman et al. 1997] lay out a framework for applying slicing to coupling and consider two small example programs. No significant empirical evaluation is conducted. In their approach, inflow and outflow are computed using slicing: the flow into $f$ is captured by identifying definitions outside $f$ contained in the slice taken with respect to principal variables of $f$, while outflow of $f$ is captured by identifying components outside $f$ whose slice includes a principal variable of $f$. 
The coupling between two modules can be computed by restricting the "components outside $f$ " to components from some other module $g$. Harman et al. observe that the slice-based definition provides a more precise measure of dependency than information flow, since the presence in the slice of a single node outside the body of $f$ indicates an information flow from $g$ to $f$. However, as well as detecting such a flow, this approach can assess the 'amount' of information flow (i.e., its 'bandwidth') by determining precisely how much of the slice lies outside the body of $f$. The slice-based measurement is therefore more precise in describing the level of information flow than measurements which merely detect its presence.

More formally, Harman et al. define the flow between modules $f$ and $g, F F_{(f, g)}^{p}$, as the ratio of the number of elements from $f$ included in interprocedural slices with respect to the principle variables of $g$ to the number of elements in $f$. Coupling is then computed as a normalized ratio of the flow in both directions:

$$
\operatorname{coupling}(f, g)=\frac{F F_{(f, g)}^{p} \times \text { length }(f)+F F_{(g, f)}^{p} \times \operatorname{length}(g)}{\text { length }(f) \times \operatorname{length}(g)}
$$

This is generalized to the coupling for a given a module, which is computed as the weighted average of its computed values with all other modules:

$$
\text { coupling }(f)=\frac{\sum_{i=0} \operatorname{coupling}\left(f, g_{i}\right) \times\left|g_{i}\right|}{\sum_{i}\left|g_{i}\right|}
$$

\subsection{Statistical Tests}

Two statistical tests are used to correlate the data. First, Pearson's linear correlation is used to quantify the relation between metrics. Such correlations measure linear associations between variables. Pearson's test assumes that the errors are normally distributed and have constant variance; however, the inferences do not depend crucially on the normality assumption [Ott and Longnecker 2001]. The output is a correlation coefficient, reported as the value $R$, and the coefficients of a linear model. The statistical significance of $R$ can be summarized as

$$
\begin{aligned}
& 0.8 \text { - } 1.0 \text { strong association } \\
& 0.5-0.8 \text { moderate association } \\
& 0.0 \text { - } 0.5 \text { weak or no association }
\end{aligned}
$$

A negative value indicates an inverse correlation. Furthermore, the value of $R^{2}$ represents the proportion of the variation in the response variable that is accounted for by the variation of the explanatory variables. For the effect of $X, Y$, and $Z$ on $A$, the resulting model coefficients, $m_{i}$, belong to the linear equation

$$
A=m_{1} X+m_{2} Y+m_{3} Z+b .
$$

Second, the Spearman test does not attempt to construct a linear correlation between the two variables, but merely gives a correlation coefficient which indicates whether increases in one variable are accompanied by increases in the other. The presence of a Pearson correlation is normally accompanied by the presence of a Spearman correlation. However, it is possible for data to have a strong Spearman correlation without having a strong Pearson correlation. When this happens, it gives some evidence that there may be a non-linear increase in the dependent variable corresponding to increases in the independent variable. 
Spearman's test is a non-parametric test and thus makes no assumptions about the distribution of the data. This test does assume the data comes from a random sample [Ott and Longnecker 2001]. The output is a correlation coefficient, again reported as the value $R$.

\section{THE STUDY}

This section reports results from the four empirical studies on slice-based coupling and cohesion metrics. First, it presents some information on the subject programs studied. This is followed by a the first study, which considers quantitative and qualitative comparisons between the slice-based metrics. The goal here is to determine which metrics measure unique aspects of the code. This first study also compares the slice-based metrics with lines of code in order to determine if the slice-based cohesion and coupling are simply proxies for size-based metrics. This is followed by the two longitudinal studies of the cohesion metrics. These two studies provide a context for the third study which considers base-line metric values. The final study considers the cohesion metric parallelism.

\subsection{Subjects}

This section introduces the $63 \mathrm{C}$ programs used as study subjects. Figure 4 presents the size of each program measured in both lines of code as counted by the unix utility wC and the number of non-comment non-blank lines of code as counted by the utility sloc. It also includes the average number of slices taken per module (i.e., the average number of principal variables). The code base represents a collection of small to medium sized stable programs, primarily open source but with a few industrial programs. The subject programs include both system utilities and application software from a variety of application domains. Two "families" of programs are included as subjects (prefixed by "cook-" and "snns-"). This allows the investigation of a common code base to be considered. Also, for the longitudinal study, multiple versions of barcode and gnugo were studied.

\subsection{Metric Comparisons}

In this section, the slice-based metrics are compared to each other and the simple size metric, lines of code, both quantitatively and qualitatively. These comparisons help provide insight into what the metrics are measuring and demonstrate that they are indeed different from code size measures. Because highly correlated metrics are effectively redundant in the information that they provide, the correlative information derived from these statistical tests can be used to identify subsets, or even a minimal subset, of the metrics that produce similar results to using all the metrics. One might, for example, favor a metric that is easy to compute if it provides similar information as a metric that is harder to compute.

The quantitative investigation applies Pearson's test to the values produced for each pair of metrics. Pearson's correlation coefficient, $R$, shows the level of linear association between the two metrics, with higher $R$ values depicting stronger correlations.

The first comparison is with the size metric lines of code (LOC). This comparison is a necessary first step as it determines if slice-based metrics are nothing more than a proxy for module size. If the metric values are not correlated to the module LOC, then they provide an alternative assessment of a module.

Figure 5 shows scatter plots that compare LOC on the $y$-axis with metrics on the $x$ axis (the plot of MaxCoverage is almost identical to that of Coverage and is not shown). Visually each scatter plot shows a high degree of randomness. The bottom of the plots have more data points because the data set contains more smaller modules than larger modules. 


\begin{tabular}{|c|c|c|c|c|c|c|c|}
\hline \multirow[b]{2}{*}{ Program } & \multicolumn{2}{|c|}{ Size (LoC) } & \multirow{2}{*}{$\begin{array}{c}\text { Average } \\
\text { slices } \\
\text { per } \\
\text { module }\end{array}$} & \multirow[b]{2}{*}{ Program } & \multicolumn{2}{|c|}{ Size (LoC) } & \multirow{2}{*}{$\begin{array}{c}\text { Average } \\
\text { slices } \\
\text { per } \\
\text { module }\end{array}$} \\
\hline & wC & sloc & & & wC & sloc & \\
\hline a2ps & 63,600 & 40,222 & 40.5 & go & 29,246 & 25,665 & 21.9 \\
\hline acct & 10,182 & 6,764 & 6.2 & ijpeg & 30,505 & 18,585 & 5.5 \\
\hline barcode & 5,926 & 3,975 & 9.4 & indent & 6,724 & 4,834 & 18.6 \\
\hline $\mathrm{bc}$ & 16,763 & 11,173 & 6.8 & li & 7,597 & 4,888 & 242.8 \\
\hline byacc & 6,626 & 5,501 & 11.8 & named & 89,271 & 61,533 & 90.0 \\
\hline cadp & 12,930 & 10,620 & 10.1 & ntpd & 47,936 & 30,773 & 32.9 \\
\hline compress & 1,937 & 1,431 & 8.8 & oracolo2 & 14,864 & 8,333 & 5.4 \\
\hline cook-c-incl & 15,553 & 9,494 & 34.0 & prepro & 14,814 & 8,334 & 5.4 \\
\hline cook-cook & 57,104 & 34,858 & 267.1 & replace & 563 & 512 & 2.5 \\
\hline cook-cook-bom & 13,005 & 7,939 & 31.1 & sendmail & 46,873 & 31,491 & 137.5 \\
\hline cook-cookfp & 13,968 & 8,526 & 31.1 & snns-batchman & 53,759 & 38746 & 182.1 \\
\hline cook-cooktime & 12,999 & 7,935 & 25.3 & snns-convert2snns & 46,457 & 33,483 & 116.5 \\
\hline cook-file-check & 11,306 & 6,902 & 28.1 & snns-ff-bignet & 46,927 & 33,822 & 114.7 \\
\hline cook-find-libs & 11,185 & 6,828 & 25.9 & snns-isnns & 46,534 & 33,539 & 116.9 \\
\hline cook-fstremp & 11,402 & 6,960 & 25.0 & snns-linknets & 47,517 & 34,247 & 114.1 \\
\hline cook-make2cook & 16,339 & 9,974 & 36.8 & snns-netlearn & 46,288 & 33,361 & 116.6 \\
\hline cook-roffpp & 12,070 & 7,368 & 33.0 & snns-netperf & 46,343 & 33,401 & 116.4 \\
\hline copia & 1,170 & 1,112 & 18.8 & snns-pat-sel & 46,273 & 33,351 & 116.6 \\
\hline csurf-pkgs & 66,109 & 38,507 & 28.2 & snns-snns $2 \mathrm{c}$ & 48,092 & 34,662 & 112.6 \\
\hline ctags & 18,663 & 14,298 & 17.3 & snns-snnsbat & 47,650 & 34,343 & 118.0 \\
\hline diffutils & 19,811 & 12,705 & 10.1 & snns-td-bignet & 46,951 & 33,839 & 114.7 \\
\hline ed & 13,579 & 9,046 & 23.7 & space & 9,564 & 6,200 & 5.9 \\
\hline empire & 58,539 & 48,800 & 18.6 & spice & 179,623 & 136,182 & 73.2 \\
\hline epwic & 9,597 & 5,719 & 10.6 & termutils & 7,006 & 4,908 & 5.6 \\
\hline espresso & 22,050 & 21,780 & 13.5 & tile-forth & 4,510 & 2,986 & 14.6 \\
\hline findutils & 18,558 & 11,843 & 8.0 & time & 6,965 & 4,185 & 5.4 \\
\hline flex2-4-7 & 15,813 & 10,654 & 14.9 & userv & 8,009 & 6,132 & 22.7 \\
\hline flex2-5-4 & 21,543 & 15,283 & 16.2 & wdiff & 6,256 & 4,112 & 6.2 \\
\hline $\mathrm{ftpd}$ & 19,470 & 15,361 & 14.4 & which & 5,407 & 3,618 & 8.5 \\
\hline gcc.cpp & 6,399 & 5,731 & 12.8 & wpst & 20,499 & 13,438 & 8.0 \\
\hline gnubg & 10,316 & 6,988 & 9.6 & & & & \\
\hline gnuchess & 17,775 & 14,584 & 31.8 & Sum & $1,752,962$ & $1,240,684$ & $2,9665.7$ \\
\hline gnugo & 81,652 & 68,301 & 34.4 & Average & 27,825 & 19,693 & 47.1 \\
\hline
\end{tabular}

Fig. 4. The Subject Programs Studied

To expand the lower range, the plot comparing LOC with Coverage is repeated using a $\log$ scale for LOC. Again the randomness is evident. This is confirmed by the statistics in Figure 6, which include output from the Pearson and the Spearman tests. The Spearman (a non-parametric) test is used as a check for the Pearson test as the normality assumption of the Pearson test is violated to varying degrees in the data. Both tests indicate that LOC has little-to-no association with the data provided by the slice-based cohesion metrics (all $R$ values are considerably less than 0.50 ).

The percent variance of the dependent variable explained by the independent variable is seen in the columns labeled $R^{2}$. In short, modules size does not explain the metric values; thus, they are not simply proxies for LOC. With the exception of Tightness, all the $R$ values are statistically significant. For Tightness $p=0.465$.

Knowing that the metrics are not proxies to LOC, the next test compares them head-to- 

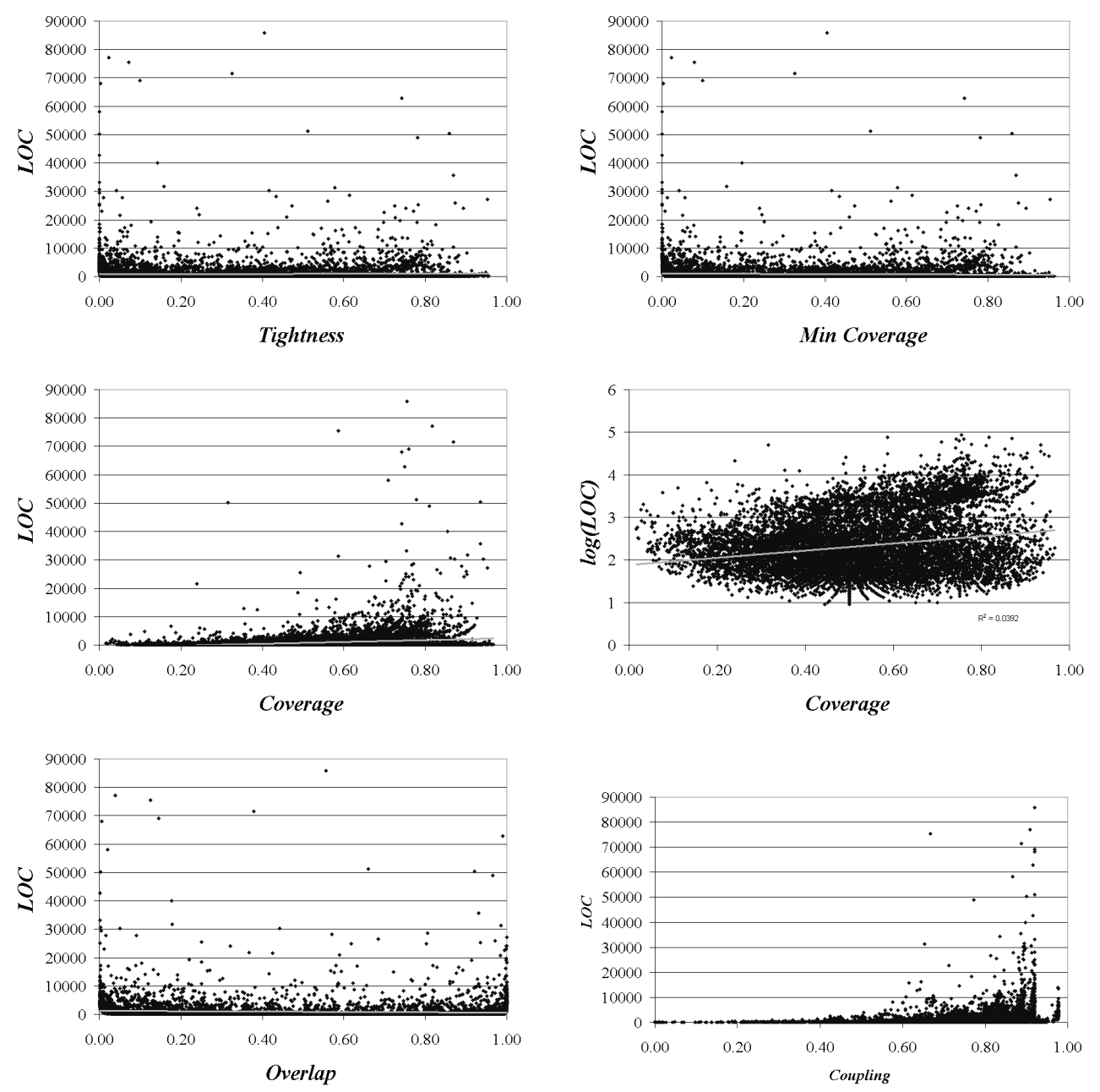

Fig. 5. Metric comparisons with Lines of Code. The middle right chart repeats comparison with Coverage using a log scale on the $y$-axis. Trend lines, which are not statistically significant, appear in grey.

\begin{tabular}{|l|r|r|r|r|}
\hline \multicolumn{4}{|c|}{ Lines-of-Code Correlations } \\
\hline \multirow{2}{*}{$x$} & \multicolumn{2}{|c|}{ Pearson } & \multicolumn{2}{c|}{ Spearman } \\
\cline { 2 - 5 }$x$ & \multicolumn{1}{|c|}{$R$} & \multicolumn{1}{|c|}{$R^{2}$} & \multicolumn{1}{c|}{$R$} & $R^{2}$ \\
\hline Tightness & 0.005 & 0.000 & -0.107 & 0.011 \\
MinCoverage & -0.046 & 0.002 & -0.224 & 0.050 \\
Coverage & 0.242 & 0.059 & 0.157 & 0.025 \\
MaxCoverage & 0.120 & 0.014 & -0.042 & 0.002 \\
Overlap & 0.069 & 0.005 & -0.043 & 0.002 \\
\hline Coupling & 0.211 & 0.045 & 0.044 & 0.020 \\
\hline
\end{tabular}

Fig. 6. Pearson and Spearman $R$ and $R^{2}$ values comparing the metrics vs. LOC. All p-scores are $<0.0001$ except Tightness where $p=0.465$. 


\begin{tabular}{|l|l|c|c|c|c|}
\hline \multirow{2}{*}{\multicolumn{2}{|c|}{$y$}} & \multicolumn{2}{c|}{ Pearson } & \multicolumn{2}{c|}{ Spearman } \\
\cline { 3 - 6 } \multicolumn{1}{|c|}{$y$} & $R$ & $R^{2}$ & $R$ & $R^{2}$ \\
\hline MinCoverage & Tightness & 0.977 & 0.955 & 0.981 & 0.962 \\
Overlap & Tightness & 0.916 & 0.839 & 0.944 & 0.891 \\
MaxCoverage & Coverage & 0.793 & 0.628 & 0.909 & 0.980 \\
Overlap & MinCoverage & 0.896 & 0.803 & 0.779 & 0.607 \\
\hline Coverage & Tightness & 0.625 & 0.391 & 0.541 & 0.292 \\
Coverage & MinCoverage & 0.602 & 0.362 & 0.532 & 0.283 \\
\hline Tightness & MaxCoverage & 0.379 & 0.144 & 0.316 & 0.100 \\
MinCoverage & MaxCoverage & 0.367 & 0.135 & 0.318 & 0.101 \\
Coverage & Overlap & 0.356 & 0.126 & 0.333 & 0.111 \\
MaxCoverage & Overlap & 0.154 & 0.024 & 0.131 & 0.017 \\
\hline
\end{tabular}

Fig. 7. Pearson and Spearman $R$ and $R^{2}$ values for head-to-head metric comparisons. All p-scores are $<0.0001$.

head. Figure 7 displays the results ordered by descending $R$ value; $R^{2}$ is also provided. As with the LOC comparisons, Spearman's test was also applied and yields similar results (actually slightly stronger in places). For each model the results are statistically significant with $p<0.0001$.

Three interesting relationships highlight Figure 7. First, the top of the table is dominated by the pair-wise comparisons between MinCoverage, Tightness, and Overlap, which show the strongest correlations. Second, of all the metrics, MaxCoverage has the least correlation with any of the others. Finally, Overlap is the most varied, having a strong linear relationship with Tightness and MinCoverage, but no linear relationship with Coverage and MaxCoverage.

Figures 8, 9, and 10 plot example comparisons from the three ranges. For example, in Figure 8, the high correlation between MinCoverage and Tightness is evident in the upper left plot. (In these plots, a perfect correlation with $R=1.0$ would appear as a solid line having the equation $y=x$.) Also evident in the figure is the strong linear correlation that Overlap has with both MinCoverage and Tightness. An explanation for this is that Tightness and Overlap are both dominated by $\left|S L_{\text {int }}\right|$. Due to the (very) strong correlation between Tightness and MinCoverage, MinCoverage and Overlap are transitively related.

The two graphs in Figure 9 show weak linear associations. It is interesting to note that these two graphs each appear to include two distinct populations. Consider the comparison of Tightness and Coverage. One population would show a strong linear correlation. It appears as a downward bulge from the line $y=x$ centered at about $(0.60,0.60)$. The other would show no correlation. It appears as an upward bulge from the line $y=0$ centered at about $x=0.40$. While several explanations for the bi-modal nature of the data were considered, none have, thus far, proved satisfactory.

Finally, Figure 10 shows two graphs for comparisons showing no linear correlation, which is visually obvious. Because highly correlated metrics are effectively redundant in the information they provide, a set of metrics that are not correlated are potentially more revealing in the sense that they provide complimentary rather than redundant information. For example, it is clear from Figure 10 that Overlap and MaxCoverage provide two distinct views and each could potentially be useful in its own way.

This subsection concludes by considering the relationship between coupling and the cohesion metrics. First off, Figure 11 shows two example charts comparing coupling and the cohesion metrics for each module. The charts for Tightness and MinCoverage are 

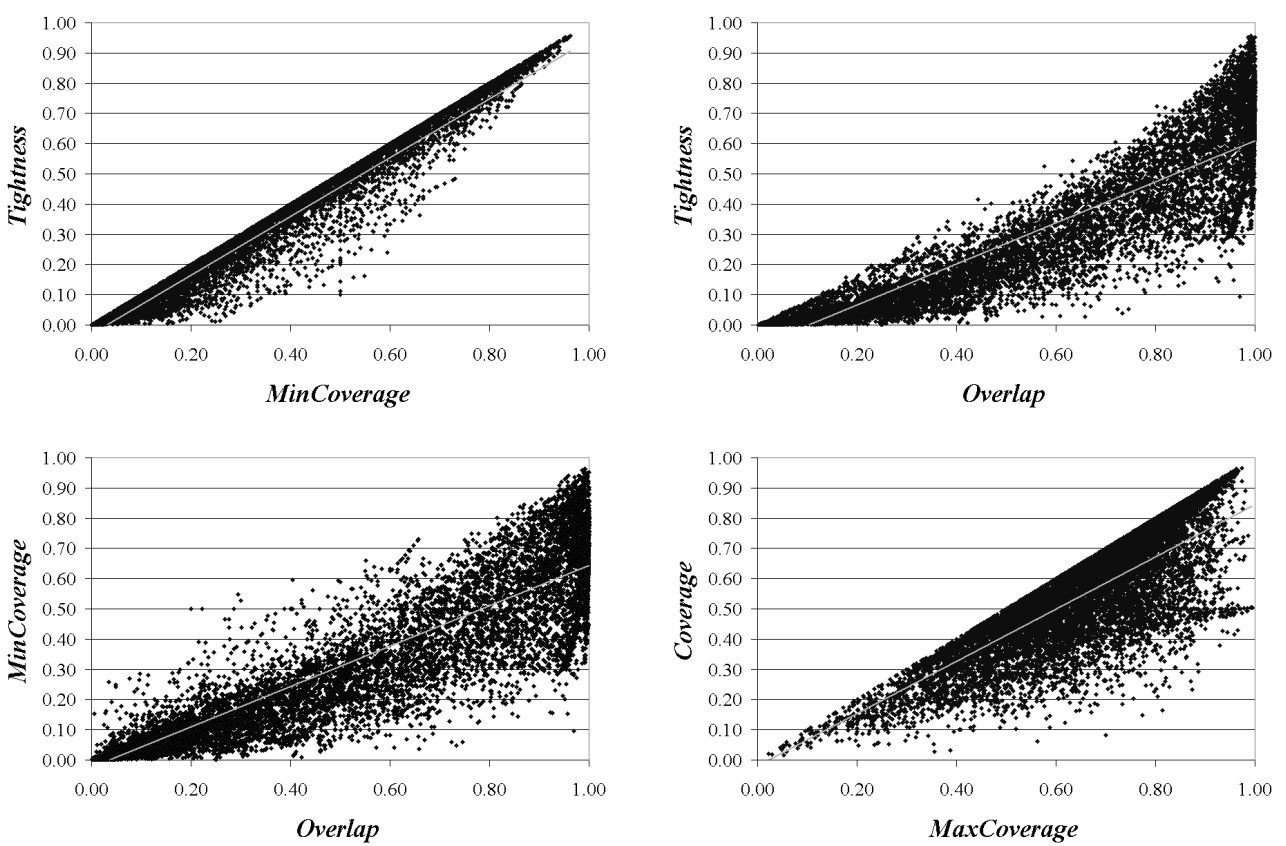

Fig. 8. Example comparisons showing strong linear correlation. Trend lines appear in grey.
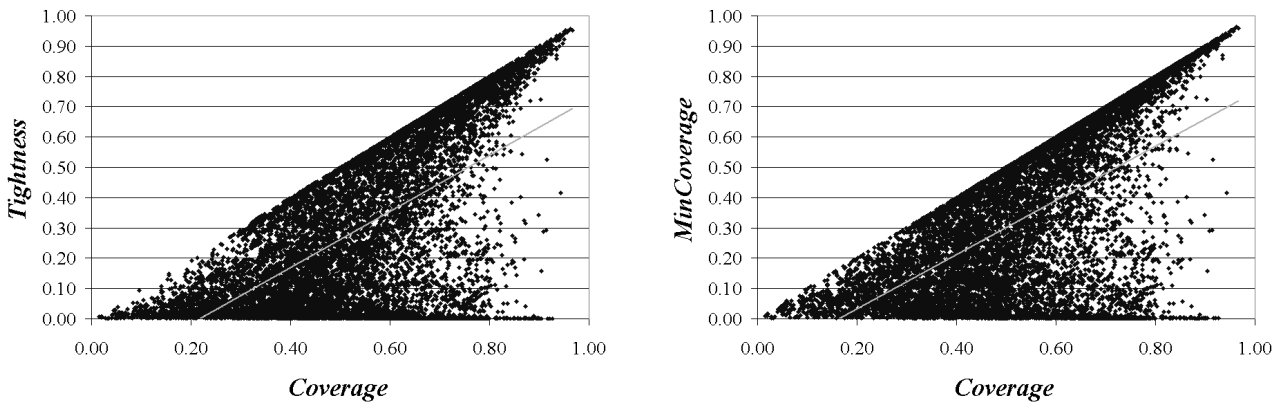

Fig. 9. Comparisons showing weak linear correlation. Trend lines appear in grey.

similar to that of Coverage with the "cloud" shifted to the left. The chart for MaxCoverage is likewise similar with the "cloud" shifted to the right. The plot for Overlap is different from the rest and shows the most random looking data. Statistically, the value of coupling has a rank correlation with Coverage and Overlap which indicates that $12 \%$ and $10 \%$ of the variation in these metrics is explained by coupling, respectively. For all the other metrics this is less than $1 \%$.

Ideally, modules would have high cohesion and low coupling. Visually, on a scatter chart with cohesion on the $x$-axis and coupling on the $y$-axis, this corresponds to the lower right of the chart. Consequently the upper left is the least desirable place for a module to appear. Using the mean coupling and cohesion values, a scatter chart can be split into four 

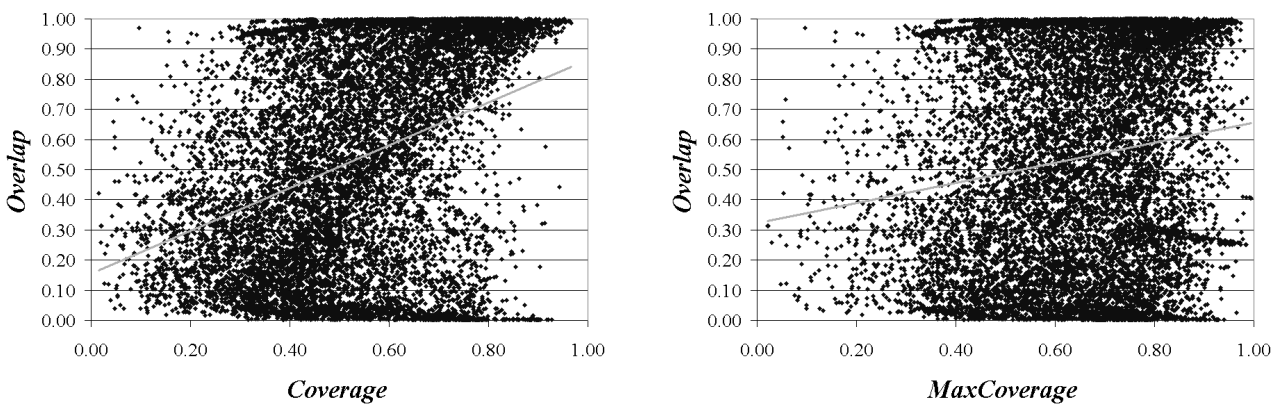

Fig. 10. Example comparisons showing no linear correlation. Trend lines are not statistically significant.
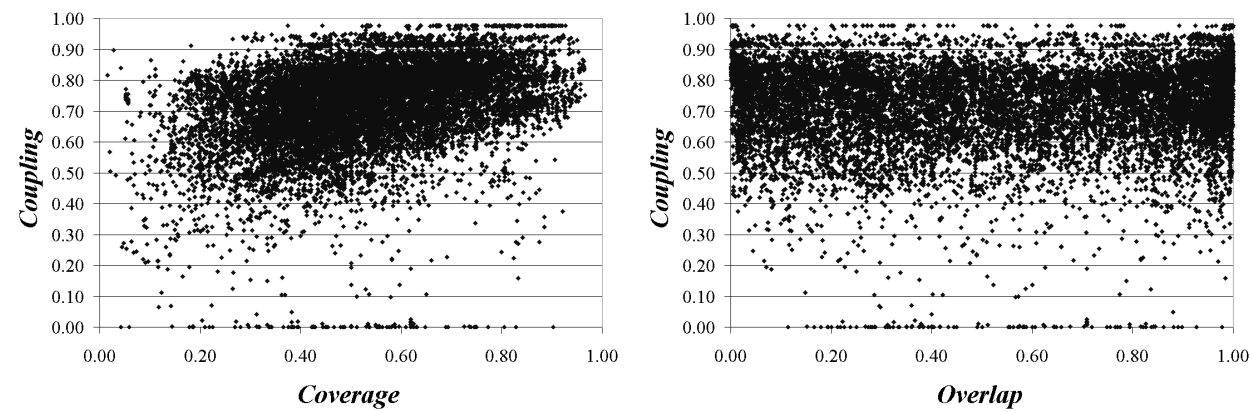

Fig. 11. Two example charts comparing coupling with cohesion

\begin{tabular}{|c|c|c|c|c|c|c|c|c|}
\hline \multirow{2}{*}{$\frac{\text { Coupling vs }}{\text { Tightness }}$} & \multicolumn{2}{|c|}{$\begin{array}{c}++(\text { Good }) \\
\text { Cohesion }>\text { mean } \\
\text { Coupling }<\text { mean }\end{array}$} & \multicolumn{2}{|c|}{$\begin{array}{c}++- \\
\text { Cohesion }>\text { mean } \\
\text { Coupling }>\text { mean }\end{array}$} & \multicolumn{2}{|c|}{$\begin{array}{c}-+ \\
\text { Cohesion }<\text { mean } \\
\text { Coupling }<\text { mean }\end{array}$} & \multicolumn{2}{|c|}{$\begin{array}{c}-(\mathrm{Bad}) \\
\text { Cohesion }<\text { mean } \\
\text { Coupling }>\text { mean }\end{array}$} \\
\hline & 3546 & $15 \%$ & 6850 & $29 \%$ & 5267 & $23 \%$ & 7653 & $33 \%$ \\
\hline MinCoverage & 3952 & $17 \%$ & 6970 & $30 \%$ & 4861 & $21 \%$ & 7533 & $32 \%$ \\
\hline Coverage & 3025 & $13 \%$ & 8551 & $37 \%$ & 5788 & $25 \%$ & 5952 & $26 \%$ \\
\hline MaxCoverage & 3165 & $14 \%$ & 9131 & $39 \%$ & 5942 & $25 \%$ & 5078 & $22 \%$ \\
\hline Overlap & 4380 & $19 \%$ & 7427 & $32 \%$ & 4426 & $19 \%$ & 7083 & $30 \%$ \\
\hline Average & 3614 & $15 \%$ & 7786 & $33 \%$ & 5257 & $23 \%$ & 6660 & $29 \%$ \\
\hline
\end{tabular}

Fig. 12. Counts from the four quadrants.

quadrants. The 'good' quadrant is the lower right where cohesion is greater than its mean and coupling is less than its mean. The 'bad' quadrant is the upper left. The two remaining quadrants have good cohesion but poor coupling, labeled +- , and poor cohesion but good coupling, labeled -+. Higher quality code should have a high number of modules in the good quadrant and a low count in the bad quadrant. For each metric, these counts are shown in Figure 12.

Many code metrics are essentially proxies for the size of a module [Gyimthy et al. 2005]. The results of this first study are important because they show that slice-based cohesion and 
coupling are not simply such proxies. Furthermore, the head-to-head comparisons allow a user to select metrics with distinctive expressive ability. They also inform an engineer when a easy to compute metric can be used in place of others. For example, it is perfectly acceptable to use MinCoverage in place of Tightness if MinCoverage is easier to compute in a given software development environment.

\subsection{Longitudinal Studies}

Having demonstrated that the metrics differ from LoC and investigated their relations to one another, this section follows a recommendation of Ott and Thuss [Ott and Thuss 1989] who suggest an investigation into the sensitivity of slice-based cohesion metrics to subsequent releases of a program. These studies show that slice-based metrics have the potential to quantify the deterioration in cohesion that a program often undergoes as it ages. Thus one goal of this section is to validate the metrics use in future projects by examining their quantitative behavior in specified situations and determining if the metrics preserve the ordering desired. This section presents two such longitudinal studies of the slice-based cohesion metrics and slice-based coupling: the first zooms in on a sequence of minor releases for barcode and the second spans several major releases of gnugo. The data suggests that slice-based metrics potentially quantify the deterioration in cohesion that a program typically undergoes as it ages.

The results are summarized in Figures 13 and 14. Note that the metrics Tightness, MinCoverage, Coverage, and MaxCoverage are ratios of a particular slice size (or set of slices) to the length of the module. Based on their definitions, the value of Tightness is always the smallest, followed by MinCoverage, Coverage, and finally MaxCoverage. Thus, these four metrics are always presented in this order. Overlap is an average percentage of common vertices within a module. It is not mathematically bounded between any of the other metrics. To begin with, Figure 13a shows growth trends for barcode. These include the significant growth in module size, and the less dramatic growth in slices per module and $\left|S L_{\text {int }}\right|$. Size growth without growth in $\left|S L_{\text {int }}\right|$ indicates the addition of "unrelated code" to the modules of the program. A typical maintenance problem.

To understand how to interpret trends in these graphs, consider an idealized target program in which each module contains a single "thought." Progress towards such a target would appear as a convergence between module size and $\left|S L_{i n t}\right|$. Thus, the divergence in Figure 13a suggests deterioration of program cohesion and continuity. In other words, software maintenance appears to be deteriorating the quality of barcode.

Figure 13b shows average metric values for the five metrics. The bold line is Overlap, which can run contrary to the other metrics. In this graph, there is a visible negative slope among all of the metrics. With the exception of Overlap, all of the metrics indicate the maximum cohesion within the first or second release and continue to fall thereafter. The deterioration (negative slope) is not very pronounced as only minor releases are considered; nonetheless, it indicates that, based on the metrics, code quality is declining.

Like barcode, gnugo's average module size (Figure 14a) exhibits general growth over the span of releases considered. These characteristics are more pronounced with gnugo as the span of releases includes two major releases. It is interesting to note that the only significant decline in average module size accompanies the major release gnugo 3.0.

The only other decline is in the very next revision (perhaps the completion of a reengineering effort). Immediately following this, gnugo experiences its largest growth over the considered releases. Gnugo under went "objectification" with version 3.0, which is also 


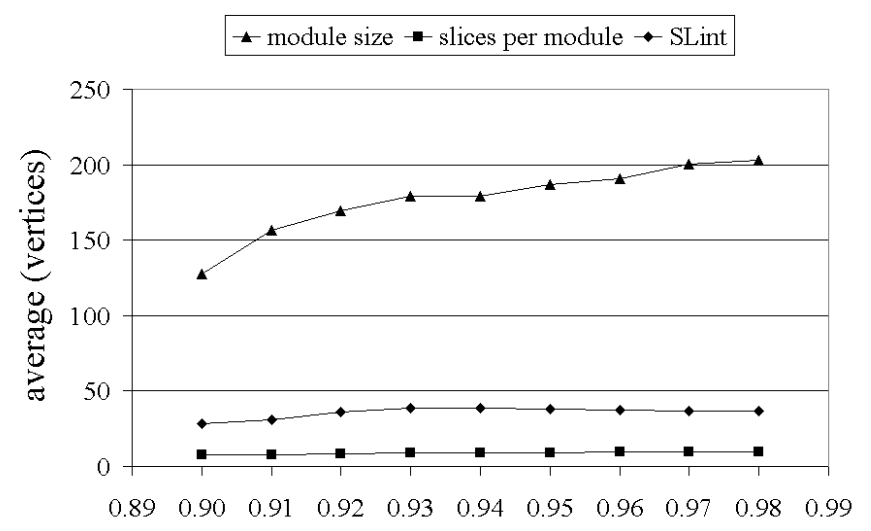

barcode version

(a) Growth

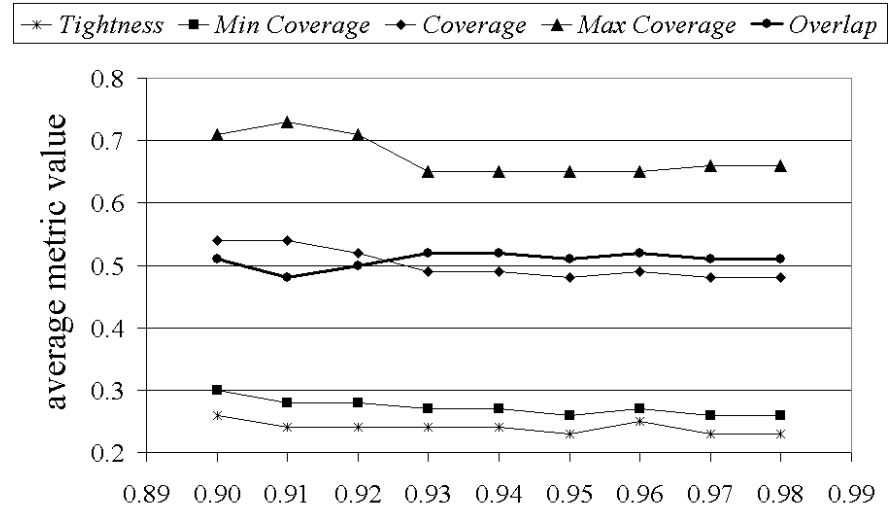

barcode version

(b) Metric Values

Fig. 13. Longitudinal study of barcode.

interesting because it is the only instance of convergence between module size and $\left|S L_{\text {int }}\right|$. This supports the notion that more thought and effort goes into a major release as opposed to seemingly atrophic minor releases.

The graph of metric values for gnugo (Figure 14b) shows a clear negative trend among all the metrics. MinCoverage, Coverage, and MaxCoverage are all in a state of constant decay with the exception of gnugo 3.0. Similarly, Tightness only experiences improvement for the major releases gnugo 2.0 and gnugo 3.0. With the exception of Overlap, all of the metrics experience their maximum value within the first or second release and continue to fall thereafter.

The above data suggests that slice-based metrics have the potential to quantify the deterioration in cohesion that a program often undergoes as it ages. In other words, it is possible to validate the metrics use in future projects by examining their quantitative be- 


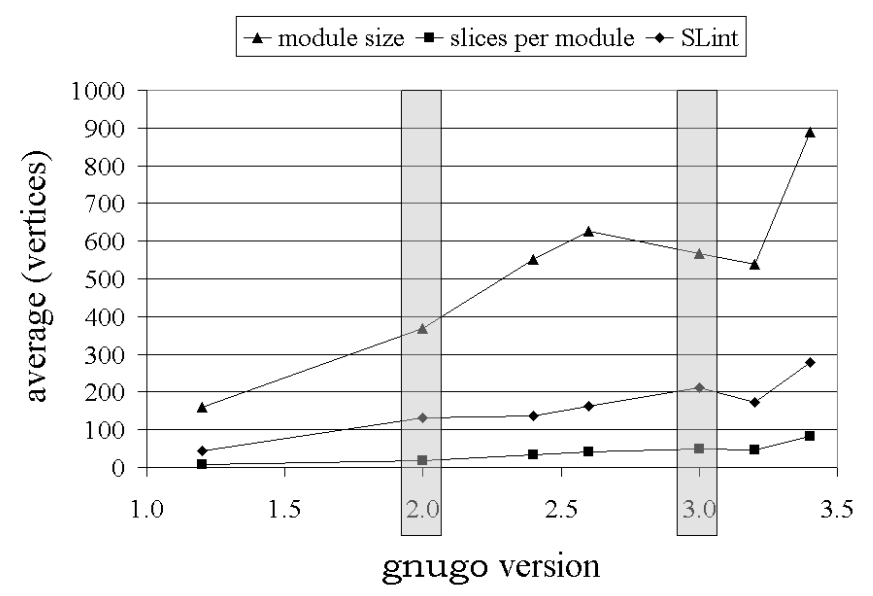

(a) Growth

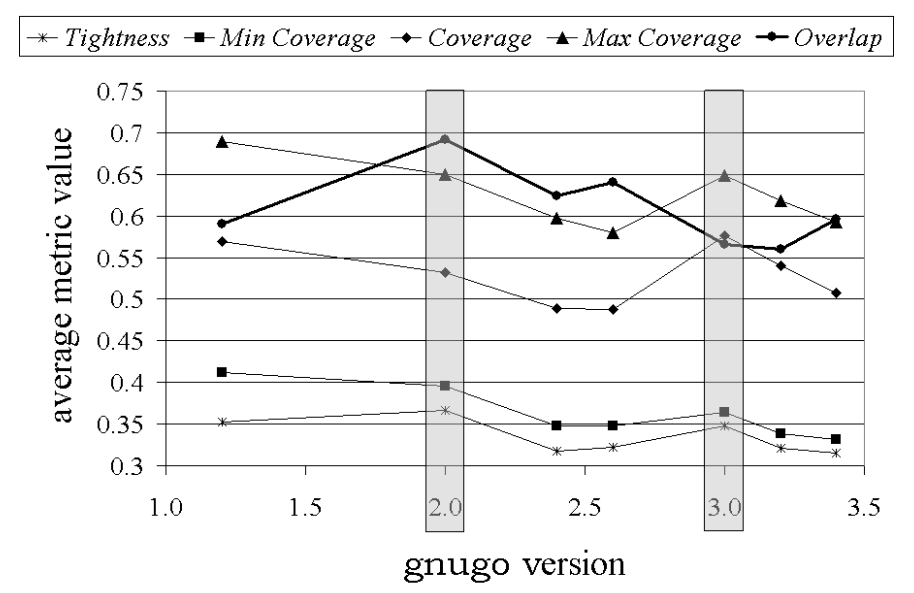

(b) Metric Values

Fig. 14. Longitudinal study gnugo. Major releases are shaded.

havior in specified situations and determining if the metrics preserve the ordering desired. Inspection of the source code for the different versions of gnugo reveal that this, in fact, occurs. The inspection uncovered the expected patching taking place between the minor releases. In contrast, the major releases clearly were given more thought-especially gnugo 3.0, where the source is given a significant overhaul. These findings are encouraging as all the metrics, except Overlap, reflect this dramatic improvement.

Switching from cohesion to coupling, Figure 15 shows the coupling data for barcode and gnugo. Barcode shows some early fluxuations, but then settles down to a slightly increasing (worsening) trend. The objectification that gnugo 3.0 underwent may account for the spike in coupling. Particularly if significant global variables were introduced temporarily and then removed in gnugo 3.2. A similar, but less pronounces peak occurs with versions 2.0 and 2.4. Thus, opposite to the cohesion metrics, improvement in coupling 

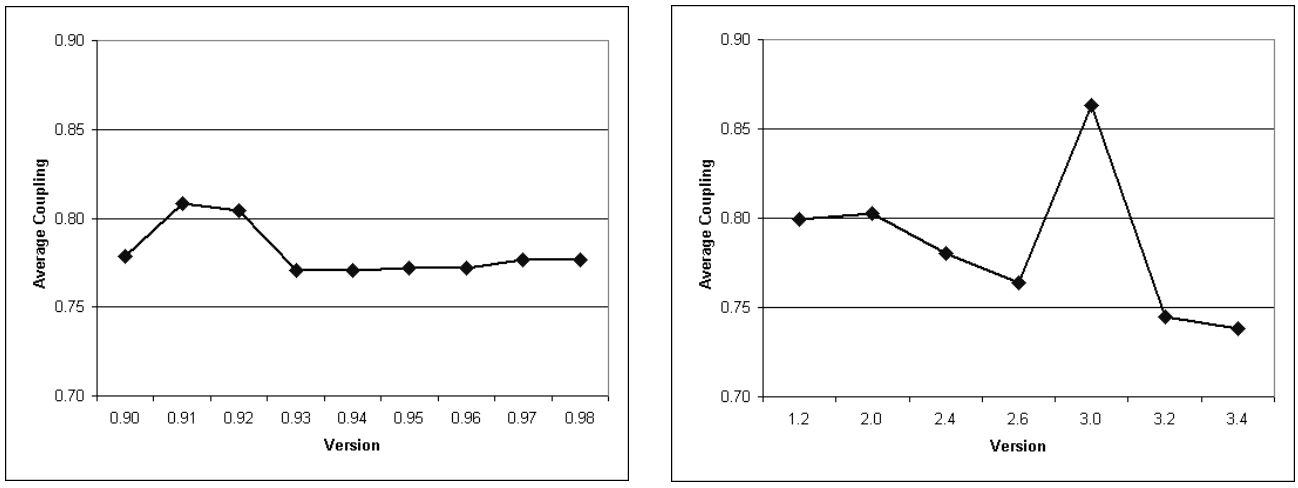

Fig. 15. Longitudinal Coupling Data

occurs with minor releases.

The next section considers base-line values for the metrics. Looking ahead, the coupling values for barcode and gnugo are both slightly higher than the base-line value except for the final version of gnugo. An informal inspection of the code for barcode and gnugo and several other subjects does not suggest that barcode and gnugo have abnormally high coupling. While comparing two different programs one has to allow for some variance due to problem domain, programming style, etc., the trends seen when comparing multiple versions of a single program are the true values of the two longitudinal studies.

\subsection{Base-Line Metric Values}

Having empirically compared the metrics head-to-head and having considered the two longitudinal studies, it is now reasonable to consider base-line metric values. Base-line values make it possible to separate aberrant functional behavior from that which is "normal"; thus, the initial goal of this study was to generate base-line values for the coupling metrics shown in Figure 1 and slice-based coupling. These values provide empirical reference values for the slice-based cohesion metrics studied by Ott and Thuss [Ott and Thuss 1993] in addition to slice-based coupling proposed by Harman [Harman et al. 1997].

Summary statistics based on the data for all 22,651 modules (without regard for source program) are given in Figure 16. The averages given provide expected values for the slicebased metrics. The standard deviations illustrate the dispersion of the data. For example, in the case of Overlap, the standard deviation of 0.3441 says that most of the Overlap points fall within a range of size 0.6882 centered about the mean, or roughly $70 \%$ of all possible values. This wide dispersion indicates that the Overlap metric is very sensitive. Conversely, MaxCoverage is the least sensitive metric. Its standard deviation of 0.1583 indicates that MaxCoverage's values is dispersed over approximately $30 \%$ of the range $[0.0,1.0]$. One use of this observation is its implication that outlier values of MaxCoverage are more significant than those of Overlap.

The confidence intervals, calculated at the $95 \%$ confidence level, demonstrate that the sample averages shown in Figure 16 are good representatives for the population of all programs. This assumes that the programs studied represent a sufficiently random sample of the population of all programs. For example, it can be said with $95 \%$ confidence that, for the population of all programs, the average value of Tightness falls between 0.2923 and 


\begin{tabular}{|c|c|c|c|c|}
\hline & \multirow[b]{2}{*}{ Average } & \multirow{2}{*}{$\begin{array}{c}\text { Standard } \\
\text { Deviation }\end{array}$} & \multicolumn{2}{|c|}{$95 \%$ confidence interval } \\
\hline & & & from & to \\
\hline Size (vertices) & 915. & 2399. & & \\
\hline Vo & 87. & 143. & & \\
\hline$\left|S L_{i n t}\right|$ & 273. & 1067. & & \\
\hline Tightness & 0.2956 & 0.2551 & 0.2923 & 0.2989 \\
\hline Min Coverage & 0.3342 & 0.2567 & 0.3310 & 0.3375 \\
\hline Coverage & 0.5365 & 0.1726 & 0.5343 & 0.5387 \\
\hline Max Coverage & 0.6425 & 0.1583 & 0.6404 & 0.6445 \\
\hline Overlap & 0.5367 & 0.3441 & 0.5323 & 0.5411 \\
\hline Coupling & 0.7281 & 0.1578 & 0.7260 & 0.7302 \\
\hline
\end{tabular}

Fig. 16. Metric averages for over all 22,651 total modules.

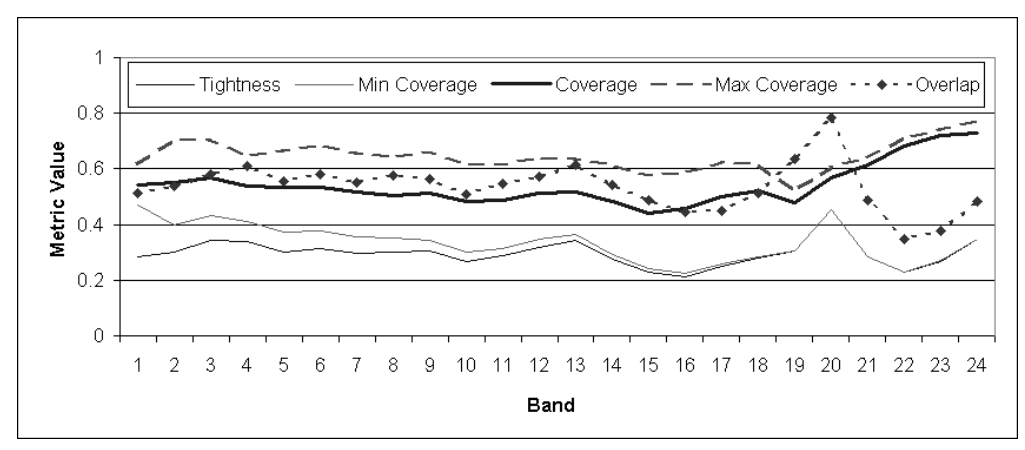

Fig. 17. Metric values by module size (grouped into 1000 wide bands).

0.2989. The other metric averages exhibit similarly small confidence intervals and thus the values from Figure 16 provide good base-line values for the five slice-based cohesion metrics studied as well as slice-based coupling.

Furthermore, while considering all outliers is not feasible, the functions having the extreme metric values for each of the five cohesion metrics were extracted and examined. Trends and insights from these are now considered. To begin with, the main function of sendmail has the lowest value of MinCoverage and Tightness. Sendmail's main is 1115 LoC. It does all the initialization which leads to many small independent slices. Having lots of slices means the module is relatively "large" while having (at least) one small slice makes MinCoverage low. The multitude of small slices makes $S L_{\text {int }}$ small; thus, leading to low Tightness value as well. The lowest values for Coverage and MaxCoverage are also from functions responsible for initialization.

The function put_ARTMAP_in_pattern from learn_f.c of snns has the lowest Overlap value. Unlike the function that performed initialization (which is expected to have low cohesion) this is an excellent example of a metric highlighting a potential place for refactoring. This function adds two "patterns" to a network. The two patterns are treated complexly and independently in the code. As a result $S L_{\text {int }}$ is small, but the individual $S L_{i}$ 's are not, leading to low Overlap. Refactoring to improve the value of Overlap would replace this function with a function to insert one pattern. The new function would then be 
called twice.

Those modules having the highest metric values include MODI_quickprop also from learn_f.c of snns. This function is a good example of a single thought function, which, as its header comment states "modifies the network at the end of each epoch". The outputs are all related to all the inputs of the computation, which leads to high Tightness. This module also has the highest MinCoverage. For the other metrics, similar single thought codes receive the highest value.

Finally, aggregating metric values over the wide range of model sizes considered may obscure patterns related to size. To address this possibility, the modules were sorted based on size and then gathered into 1000 module wide bands (e.g., the 1000 smallest models, followed by the next 1000 smallest, etc.) Figure 17 shows the metric values for each band. Visually, all five metrics hover around their mean with two exceptions. First, at the right of the chart where, for large modules sizes, there is more variation ending in an increase in metric value. Second, MinCoverage has a clear downward trend in the beginning. This occurs because all slices include a small fixed number of vertices. In very small modules, this has a noticeable effect on MinCoverage.

Some other patterns occur. For example, in large modules, MinCoverage and Tightness become almost identical. This is because the two are equal when the smallest slice is the intersection slice $\left(S L_{i n t}\right)$, which is more likely to occur in larger modules that have a lot of slices. A similar pattern exists between Coverage and MaxCoverage which hover around their means except at the high end where some rather large slices pull MaxCoverage and Coverage up. This is caused by a few large modules, often machine generated (e.g., by yacc), that have only a few highly interdependent slices.

Statistically, the banded metrics data is not normally distributed; thus Spearman's nonparametric test was used to look for associations between size and metric value. The $R^{2}$ values, all five of which are statistically significant $(p<0.0001)$, range from 0.048 for MinCoverage to 0.002 for MaxCoverage, indicating that the (rank of a) size has very little influence on the (rank of a) metric score.

Although, two of the Pearson test's assumptions (that the errors are normally distributed and have constant variance) are violated to varying degrees in the data, it is still possible to consider the percent variance. However, no statistical confidence can be placed on the result. The largest variance, 0.395 for MinCoverage, seems plausible given the general downward trend of MinCoverage seen in Figure 17. The next largest, Coverage at 0.182, indicates that $18 \%$ of the variance in Coverage is accounted for by size. The values for the remaining metrics are all under $10 \%$.

\subsection{Parallelism}

Parallelism is singled out because it produces multiple values for each module and is thus difficult to summarize and compare with metrics producing a single value. It is intended to measure the amount of code that is independent (can be executed in parallel). The definition is parameterized by $\tau$ which captures permissible overlap in the slices. Thus, with $\tau$ set to zero no overlap is allowed. With slightly higher values, limited shared code (e.g., the initialization of a shared variable) is permitted.

Being parameterized by $\tau$, Parallelism takes on multiple values (one value for each value of $\tau$ as shown in Figure 1). Furthermore, the empirical data suggests that it is the least useful of the studied metrics. In essence, the data suggest that a refined definition 
may be more appropriate. For example, 18,930 of the 22,651 modules studied have the same Parallelism value for all values of $\tau$. For these modules the value of Parallelism degenerates to $\left|S L_{\text {int }}\right|$. (This assumes values of $\tau$ less than $\left|S L_{\text {int }}\right|$ are ignored. By definition, Parallelism for these values of $\tau$ is zero. In the sequel, only values for $\tau$ greater than or equal to $\left|S L_{i n t}\right|$ are considered.)

There are two primary sources of the 18,930 singletons. First, some modules simply have a single output and thus a single slice. Second, if the intersection of one slice, $S$, with all the other slices has size one, then each $S L_{i}$ will have an $S L_{j}$ (i.e., $S$ ) such that the size of $S L_{i} \cap S L_{j}$ is one. An example case in which this occurs is when a global counter is incremented by a function. When the slice on this counter's final value is independent of all the other slices of the module, all slices are included in $\operatorname{Parallelism}(M, \tau)$ regardless of $\tau$ 's values. In such cases, the parallelism that might exist between other slices of the module is masked.

Figure 18 provides three graphical views of Parallelism. Unlike the other metrics, Parallelism does not yield a value between 0.0 and 1.0. Furthermore, the possible values for $\tau$ are not necessarily the same for different modules ( $\tau$ ranges from 1 to $N_{V}$, the number of vertices in the module's PDG). In order to compare the Parallelism of different modules, both values are normalized: the value of Parallelism is normalized to a percentage of $V_{O}$, while the value of $\tau$ is normalized to a percentage of $N_{V}$.

The top graph in Figure 18 shows the average results for all 22,651 modules and for the 3,721 modules that included at least two distinct values (i.e., the non-singletons). For singleton sets, Parallelism equals $\left|S L_{i n t}\right|$. Given the large number of singletons, the "All Data" line is dominated by $\left|S L_{\text {int }}\right|$.

The line for the non-singleton values indicates significant parallelism. For example, this line includes the point $(20 \%, 77 \%)$, which indicates that, on average, $77 \%$ of all slices have no more than $20 \%$ of their module in common. At first this seems to contradict the conventional wisdom that sequential code lacks significant parallelization opportunities. An explanation lies in the rather finely grained nature of the parallelism identified by this metric (e.g., running two statements in parallel as opposed to splitting a function into two parallelizable halves).

Ignoring the 18,930 singletons, leaves 3,721 modules. Of these, 3,200 have only two distinct values. Those having 3 - 9 distinct values number 399, 86, 25, 4, 2, 3, 1, respectively, with 1 module having 13 distinct values. The last five, having the most distinct values, were used to generate the lower two charts in Figure 18. The middle chart was built from the normalized data, while the bottom chart was built from the unnormalized data. A comparison of these two charts helps to illustrate the effect of normalizations. Consider the module with 13 distinct Parallelism values (the top line of the bottom chart of Figure 18). The start of the first plateau on this line represents 10 slices that have no more than 300 vertices (about 50 statements) in common. The start of the final plateau indicates that all 86 slices have no more than 400 vertices (about 68 statements) in common.

\section{RELATED WORK}

This section considers work related to slice-based metrics with a focus on work that includes empirical data. Broader motivation for the (empirical) study of metrics in general, and cohesion metrics in particular, has been recently argued by Mens and Demeyer in their classification of approaches that use metrics [Mens and Demeyer 2002]. For example, 


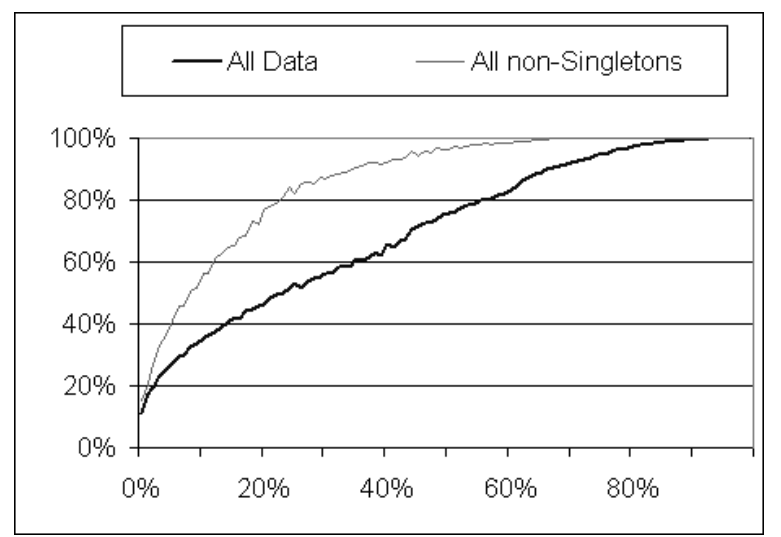

Average Parallelism values for all modules and all non-singelton modules: the $y$-axis shows the percent of $V_{O}$ and the $x$-axis the percent of $N_{V}$

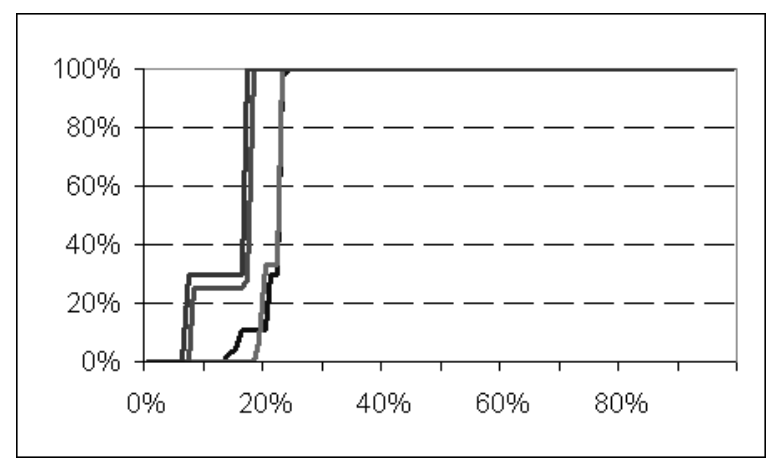

Average Parallelism values for modules with five or more distinct levels of paralelism: normalized - the $y$-axis shows the percent of $V_{O}$ and the $x$-axis the percent of $N_{V}$

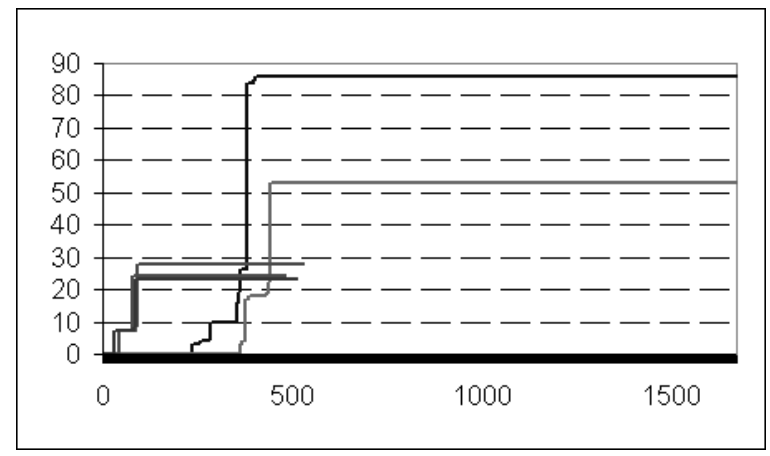

Average Parallelism values for modules with five or more distinct levels of paralelism: unnormalized - the $y$-axis shows the size of $V_{O}$ and the $x$-axis the size of $N_{V}$

Fig. 18. Three views of the Parallelism. In the first two charts the $y$-axis is the percentage of $V_{O}$ and the $x$-axis is the percentage of $N_{V}$. In the bottom chart the $y$-axis is the number of slices and the $x$-axis is the number common statement represented by $\tau$. 
Demeyer et al. describe a reverse engineering approach that combines metrics and visualization [Demeyer et al. 1999]. They purposefully chose to work with a collection of simple metrics (parameter count, number of (static) invocations, etc.). They report that the combination shows promise. However, they do question whether "a simple measurement is a[sic] sufficient to assess such [a] complex thing as software quality."

As mentioned in the introduction, slice-based coupling metrics have not been well studied. Harman et al. observe that their work "is the first to address the problem of coupling measurement using slicing" [Harman et al. 1997]. No empirical work on slice-based coupling has since been undertaken. Thus, only related work on slice-based cohesion is considered. This is done in roughly chronological order beginning with Weiser's original work, followed by the considerations of metric slices, control and data metrics, "glue" tokens, and the granularity of metrics. Finally, a study by Karstu is considered.

Weiser applied the metrics from Figure 1 to several student-written load-and-go compilers as a proof of concept [Weiser 1981]. Their further study by Ott and Thuss led to the development of the metric slice [Ott and Thuss 1993]. A metric slice on $v$ is the union of the backward slice on $v$ taken from the end of the module and the forward slice computed from every statement that defines $v$ within the backward slice. A collection of carefully chosen examples was used to motivate the use of the metric slices over traditional slices. In comparison with the slices used in Section 3, a metric slice is likely to be larger. This may mean that it contains more information and hence proves more useful, or that it includes too much of the program and hence leads to a reduction in utility. Empirical investigation is required to determine the relation between the utility of these two definitions. Neither Weiser nor Ott and Thuss undertake a significant empirical study of these metrics as it was infeasible at the time.

Bieman and Ott recast slice-based metrics in terms of two kinds of "glue" tokens [Bieman and Ott 1994]: To gain an intuition for their approach, it is sufficient to treat a "token" as an SDG vertex. Bieman and Ott define two kinds of glue tokens (in their definitions, slices are taken with respect to module outputs):

- The glue tokens of module $m$ are those tokens in two or more slices.

- The super glue tokens of module $m$ are those tokens in all slices (i.e., $S L_{\text {int }}$ ).

Glue and super-glue tokens are then used to define and study three cohesion metrics: StrongCohesion-the proportion of a module's vertices that are super-glue tokens (this is essentially the same as Tightness), WeakCohesion-the proportion of a module's vertices that are glue tokens, and Adhesiveness-the average number of slices glued together by the tokens of a module. In their work, Bieman and Ott treat only a few small code segments.

Harman et al. expand the work of Ott et al. by considering the elements that each metric is based on [Ott and Thuss 1989]. The result is a collection of "fine-grain" metrics obtained by replacing the "number of statements" with $V(e)$ the number of distinct variables in expression $e, N(e)$ the number of internal nodes in the abstract syntax tree of the expression $e, L(e)$ the number of leaves in the abstract syntax tree of the expression $e$, and $T(e)$ the number of tokens in the concrete syntax of the expression $e$. They conclude that the original statement-based metrics and those based on $V(e)$ appear "to be inappropriate as a basis for calculating [cohesion]" [Harman et al. 1995a]. Using $N(e), L(e)$, and $T(e)$, which capture a finer level of granularity, the metrics produce the intuitively expected result for a carefully chosen example. However motivational, this example is only eight lines of code. 
Finally, in his masters thesis, Karstu describes empirical work on slice-based metrics [Karstu 1994]. Like the research proposed herein, Karstu's goals include investigating the usefulness of slice-based cohesion measures for real software. (At the time of its writing, all previous studies had been based on non-production software. For example, Thuss' study was based on student Pascal programs and ignored key language features such as pointers.) Karstu's slicer had its limitations. For example, pointers were treated as regular variables. In contrast, the slicer used in the study presented herein performs extensive points-to analysis and handles the complete $\mathrm{C}$ language; thus, it does not have these limitations.

These limitations make direct comparison of results difficult. For example, Karstu obtains a wider range of values for each metric. This is unexpected given that the study from Section 3 considers over 2000 times more code. One would expect that considering more code would lead to a wider range of metric values. This is possibly an artifact of slicer inaccuracy in Karstu's study, but could also come from the homogeneity of the code studied herein. Karstu does not give numeric averages for the metrics, but visually, from charts in his thesis, the average values for Tightness and MinCoverage are about the same as those in Figure 16 while the values for the remaining metrics are smaller than those in Figure 16. This is consistent with a slicer that misses the data dependences related to pointers returning smaller slices.

\section{THREATS TO VALIDITY}

With any empirical experiment, it is important to consider threats to validity. In the absence of human subjects, only two potential threats to validity need to be considered. These are threats to internal and external validity. External validity, sometimes referred to as selection validity, is the degree to which the findings can be generalized. In this study, it is possible that the selected programs are not representative of programs in general and thus results from the experiment do not apply to 'typical' programs.

Considering 63 subject programs helps to mitigate this concern. The diversity of these programs makes it more likely that the conclusions which are made about the techniques generalize to wider classes of programs. For example, the program prepro is Fortran-esc in its use of arrays. In contrast, several other programs make heavy use of function pointers. The program ed is rather tightly knit and "single minded". In contrast, the program acct contains many different (related) computations. However, as most of the programs are open-source programs, it remains possible that non-open-source programs (i.e., programs built in any radically different culture) will exhibit different behavior. Finally, while all programs share a certain amount of commonality in their structure, the farther away a program is from the 63 programs studied herein, the more likely the chance that its metric values do not conform to those observed in the 63 programs.

Internal validity is the degree to which conclusions can be drawn about the causal effect of the independent variable on the dependent variable. In this experiment, the only serious threat comes from potential for faults in the slicers. Other common forms of internal validity, for example, construct validity (the degree to which the variables used in the study accurately measure the concepts they purport to measure), are not an issue, because the variable measured (slice size), can be measured with high precision. It is important to

note that this study empirically investigates slice-based cohesion and coupling as defined by others [Weiser 1981; Ott and Thuss 1993; Harman et al. 1997]. This can be done with 
high accuracy. It does not study the correlation between slice-based coupling and cohesion and the coupling and cohesion as intuitively defined by a software engineer. Doing so would involve human subjects' assessment of coupling and cohesion and would clearly require the consideration of construct validity. Thus, the only serious internal validity concern comes from the assumption that tools correctly implement the slicing algorithm. In practice, the slicer might contain errors, or employ imprecise analyses (e.g., imprecise data-flow analysis or imprecise points-to analysis). To mitigate this concern, a mature slicing tool was used. This tool has been throughly tested over a period of more than ten years. This reduces the impact that implementation faults may have on the conclusions reached regarding dependence analysis.

\section{SUMMARY AND FUTURE WORK}

This paper presents a large scale empirical investigation of slice-based metrics and provides three key contributions:

-First, the paper provides a head-to-head comparison of the metrics; thus, providing a better understanding of their relationships and indicating which metrics provide similar views of a program and which provide complimentary views of a program. This study also illustrated that the metrics are not proxies for size-based metrics.

- The second result comes from the two longitudinal studies, which show that slice-based metrics can be used to quantify the deterioration that accompanies software evolution. Thus, the studied metrics have the capacity to be used at the program level to guide the effects of reverse engineering's attempt to "improve" code.

-Third, given the large code based used, the values reported herein provide good estimates of expected metric values. These values can be used at the module level to focus the attention of reverse engineers on particularly abject modules.

Finally, conducting this study has suggested several areas for future work. The most important future study addresses the pragmatic question "does a software engineer with access to slice-based metric values for the modules of a program do a better job of restructuring the program?" While the longitudinal studies provide encouraging initial evidence, with only two programs, no statistically significant results can be derived. The data does suggest that a more comprehensive study is warranted.

\section{ACKNOWLEDGMENTS}

GrammaTech provided CodeSurfer ${ }^{t m}$ upon which the implementation is based. Special thanks to Mark Harman for guidance throughout the writing of this paper and the referees (especially Referee 3 ) for their thoughtful comments and suggestions. Conversations and the first Astranet meeting and WCRE helped to focus this work. This work is supported by National Science Foundation grant CCR0305330.

\section{REFERENCES}

Bieman, J. And Kang, B. February 1998. Measuring design-level cohesion. IEEE Transactions on Software Engineering 24, 2, 111 -124.

Bieman, J. And OTt, L. 1994. Measuring functional cohesion. IEEE Transactions on Software Engineering 20, 8 (Aug.), 644-657.

Binkley, D. And Gallagher, K. 1996. Program slicing. In Advances in Computers, Volume 43, M. Zelkowitz, Ed. Academic Press, 1-50.

ACM Transactions on Software Maintenance, Vol. V, No. N, November 2007. 
Binkley, D., Horwitz, S., AND RePs, T. 1995. Program integration for languages with procedure calls. ACM Transactions on Software Engineering and Methodology 4, 1, 3-35.

Constantine, L. L. And Yourdon, E. 1979. Structured Design. Prentice Hall.

DEMEYER, S., DUCASSE, S., AND LANZA, M. 1999. A hybrid reverse engineering approach combining metrics and program visualization. In Working Conference on Reverse Engineering. 175-186.

GRAMMATECH INC. 2002. The codesurfer slicing system.

Gyimthy, T., Ferenc, R., AND SiKet, I. 2005. Emperical validation of object-oriented metrics on open source software for fault prediction. IEEE Transactions on Software Engineering 31, 10 (Oct.), 897-911.

Harman, M., Danicic, S., Sivagurunathan, B., Jones, B., And Sivagurunathan, Y. 1995b. Cohesion metrics. In $8^{\text {th }}$ International Quality Week. San Francisco, Paper 3-T-2, pp 1-14.

Harman, M., Danicic, S., Sivagurunathan, B., Jones, B., and Sivagurunathan, Y. 30 May - 2 June 1995a. Cohesion metrics. In Proceedings of the 8th International Software Quality Week(San Francisco CA), 4-T-4.

Harman, M., Okunlawon, M., Sivagurunathan, B., And Danicic, S. 17-23 May 1997. Slice-based measurement of coupling. In Proceedings of the IEEE/ACM ICSE workshop on Process Modelling and Empirical Studies of Software Evolution (Boston, Massachusetts), 28-32.

HenRY, S. AND KAFURA, D. 1981. Software structure metrics based on information flow. IEEE Transactions on Software Engineering 7, 5, 510-517.

HorwitZ, S., REPS, T., AND BINKLEY, D. 1990. Interprocedural slicing using dependence graphs. ACM Transactions on Programming Languages and Systems 12, 1, 26-61.

KARSTU, S. August 1994. An examination of the behavior of slice based cohesion measures.

LAKHOtia, A. AND Sterling, L. 1988. Composing recursive logic programs with causal join. New Generation Computing 6, 2, 211-225.

LI, L. AND OfFutT, A. J. 1994. Algorithmic analysis of the impact of changes to object-oriented software. In International Conference on Software Maintenance (ICSM'96). IEEE Computer Society Press, Los Alamitos, California, USA, Victoria, Canada, 424-433.

Mens, T. And Demeyer, S. 2002. Future trends in software evolution metrics. In Proceedings of the 4th international workshop on Principles of software evolution. ACM Press, 83-86.

OTt, L. And Thuss, J. 1989. The relationship between slices and module cohesion. In Proceedings of the Eleventh Intetnationl Conference on Software Engineering, 198-204.

OTT, L. AND Thuss, J. 1993. Slice based metrics for estimating cohesion. In Proceedings of the First Intetnationl Software Metrics Symposium, $71-81$.

Ott, R. And Longnecker, M. 2001. An Introduction to Statistical Methods and Data Analysis, $5^{\text {th }}$ ed. Duxbury Press.

Page-Jones, M. 1980. Practical Guide to Structured System Design. Prentice Hall.

Podgurski, A. AND Clarke, L. 1990. A formal model of program dependences and its implications for software testing, debugging, and maintenance. IEEE Transactions on Software Engineering 16, 8.

Redwine, S. AND RidDle, W. 1985. Software technology maturation. Proceedings of the eighth IEEE/ACM International Conference on Software Engineering, (London, UK, August, 1985), 189-200.

RePs, T. And Yang, W. 1988. The semantics of program slicing. Tech. Rep. Technical Report 777, University of Wisconsin.

WeISER, M. 1981. Program slicing. In $5^{\text {th }}$ International Conference on Software Engineering. San Diego, CA, 439-449.

Zelkowitz, M., Wallace, D., AND BinKley, D. December 1998. Culture conflicts in software engineering technology transfer. In Proceedings of the 23rd NASA Goddard Space Flight Center Software Engineering Workshop (Greenbelt, Maryland), 52-62. 